\title{
The Roles of Extracellular Purinergic Signaling in Local Acupoints In Chrono- Acupuncture Analgesia
} \author{
Nannan Liu ${ }^{1}$ and Dingjun Cai ${ }^{1 *}$ \\ ${ }^{1}$ School of Acupuncture and Tuina, Chengdu University of Traditional Chinese Medicine, China \\ ${ }^{2}$ Chongqing Mental Health Center, China \\ ${ }^{3}$ Xiangyang Hospital of Traditional Chinese Medicine, China \\ ${ }^{4}$ Xinjin Hospital of Traditional Chinese Medicine, China
}

Shiqi Huang ${ }^{1}$, Xiaoxiao Lu$^{1}$, Xiaowei Shen ${ }^{2}$, Zhengyu Zhao' ${ }^{1}$, Qizhi Zhou ${ }^{1}$, Jilan Zhao' ${ }^{1}$, Yu Wang ${ }^{3}$, Xiaohong Zhang ${ }^{4}$,

Submission: July 22, 2019; Published: August 21, 2019

*Corresponding author: Dingjun Cai, School of Acupuncture and Tuina, Chengdu University of Traditional Chinese Medicine, Chengdu, Sichuan, China

Abstract

Objective: To explore the peripheral mechanism of chrono- acupuncture with respect to purinergic signaling in local acupoints.

Methods: The Sprague Dawley (SD) rats were randomly divided into two groups. One group was injected $0.1 \mathrm{ml}$ saline as control group. Another group was established by subcutaneous injecting $0.1 \mathrm{ml}$ complete Freund's adjuvant (CFA). After modeling successfully, model rats were randomly divided into model group and acupuncture group based on the basic pain threshold. The basic pain threshold was tested by tailflick method. The extracellular ATP concentration in local acupoints was detected by high- performance liquid chromatography (HPLC) after acupunctured at different times, which were zeitgeber time 0(ZT0) (7:00), ZT4(11:00), ZT8(15:00), ZT12(19:00), ZT16(23:00), and ZT20(3:00) respectively. Based on the characteristics of the variation of pain threshold and extracellular ATP concentration, the peak phase and valley phase were selected for the further experiment. The protein expression of P2X3 receptors in skin of "Zusanli" (ST36), dorsal root ganglion (DRG) and spinal dorsal horn (SDH) were separately detected by immunohistochemistry.

Results: Time had an evident influence on pain threshold $(\mathrm{P}=0.047, \mathrm{P}<0.05)$ which indicated the pain threshold at different time points was different. The variation of pain threshold among control group, model group and acupuncture group were significantly different $(\mathrm{P}<0.01)$. In acupuncture group, the of peak value and valley value appeared at ZT8 and ZT16 respectively. Pain threshold showed complex interactions with extracellular ATP, time factor and acupuncture stimuli. The extracellular ATP concentrations were significantly different in comparison among control group, model group and acupuncture group respectively. ( $\mathrm{P}<0.01)$. As for protein expression of P2X3 receptors in skin of "Zusanli" (ST36), compared with control group, it obviously increased in model group at ZT8 and ZT16. And there was significant difference between control group and model group $(\mathrm{P}<0.01)$. In addition, compared with model group, it obviously decreased after acupunctured at ZT8. And there was significant difference between model group and acupuncture group at ZT8 $(\mathrm{P}<0.01)$, but not at ZT16 $(\mathrm{P}>0.05)$. Interestingly, the protein expression of P2X3 receptors in both DRG and SDH was consistent with that in skin of "Zusanli" (ST36).

Conclusion: Temporal variation of purinergic signaling participated in the initial mechanism of chrono-acupuncture analgesia in local acpoints. Also, that might be the base of dynamic variation of acupoints reactivity.

Keywords: Circadian rhythm; Chrono-acupuncture analgesia; Pain; ATP; P2X3 receptor

\section{Introduction}

Circadian rhythm was one of life characters. Peripheral clocks possessed by almost all cells [1]. Circadian clock could be entrainmented by external environments, such as darkness and light, the seasons, the social behaviors, acupuncture and so on [2]. circadian clock encodes key regulators of response to therapeutic treatment [3]. Various of pain syndromes not only showed circadian rhythm, but also affected the circadian rhythm of the body. Most migraine attacks begin in the early morning [4]. many patients with RA present a circadian rhythm in symptoms severity with a significant worsening in 
the morning. Additionally, studies have shown that the change of pain threshold had obvious circadian rhythm [5-6]. It has been recognized that acupuncture, as an effective means of treatment, could relieve various kinds of pain syndromes [78]. The electro-acupuncture has regulation effects on circadian rhythm of temperature and melatonin in depression rat model [9]. Therefore, the curative effect of acupuncture at different times was different. In terms of chrono-acupuncture analgesia, the studies, pay more attention to central mechanism. Compared with the normal acupuncture, premature acupuncture for the regulation of dysmenorrhea model rats' $\beta$-EP content and HSP70 expression in hypothalamus and pituitary more obvious effect. That might be related to mechanism of central analgesia [10]. However, the peripheral mechanism of chrono-acupuncture analgesia, so far, poorly understood.

Acupoints, as the base of acupuncture stimulation, were the initial taches of acupuncture therapy. The temporal specificity of acupoint reactivity is one of important influence factors of acupoint reactivity. Some studies had shown that purine and its receptors involved in acupuncture analgesia. Acupuncture could increase the concentration of extracellular purine in local acupoints of rats, and the concentration of extracellular purine reached peakvalue at $30 \mathrm{~min}$, then decreased gradually [11]. P2X3 receptor, as a member of non-selective ATP gated ion channel, participated in the conduction of peripheral painful information through binding to extracellular ATP [12]. Also, the protein and gene expression of P2X3 receptors in DRG increased in various kinds of painful animal models, accompanying with the change of ATP current [13-15]. While the gene expression of P2X3 receptors and ATP current in DRG had showed a downward trend after electro-acupuncture [16]. What's more, the extracellular purinergic signal, such as ATP, not only had its own circadian rhythm, but also showed the function of time modulation. Thus, we proposed the hypothesis that, in the peripheral mechanism, the acpoint reactivity of chrono-acupuncture analgesia might be related to the temporal changes of purinergic signaling in local acupoints.

\section{Results}

\section{The relationship among time factor, ATP \& pain threshold on chrono-acupuncture}

There were no significant differences among the weight and basic pain threshold of each rats $(\mathrm{P}<0.05)$. In control group, the pain threshold peaked at ZT0 and reached the bottom at ZT12 which entails time had a notable effect on pain threshold $(\mathrm{P}<0.05=$. After modeling, both the circadian rhythm of pain and the phase of the pain threshold had changed. compared with control group, the pain threshold of model group decreased. It showed that it peaked at ZT8 and reached bottom at ZT16. However, time had no obvious effect upon the pain threshold in model group $(\mathrm{P}=0.076, \mathrm{P}>0.05)$. After acupuncturing, compared with model group, the pain threshold of acupuncture group increased. The peak occurred at ZT8 and the valley occurred at ZT16. Time factor had a significant influence on pain threshold of acupuncture group $(\mathrm{P}<0.05)$ Table1- Table 5, Figure 1 . The interaction played a notable part in pain threshold. When compared the pain threshold among control group, model group and acupuncture group, they were significantly different $(\mathrm{P}<0.01)$ Figure 2. The temporal variation tendency of pain threshold in each group. In the control group, two valley value were at ZT4 and ZT12 respectively. The temporal variation of pain threshold in model group showed abnormal pathological changes. While, compared with model group, the pain threshold of acupuncture group showed upward and it also showed abnormal pathological changes.

Table 1: Comparison of the variation of pain threshold among three groups at different times points $n=6$ (unit: second).

\begin{tabular}{|c|c|c|c|c|c|c|}
\hline & ZT0 & ZT4 & ZT8 & ZT12 & ZT16 & ZT20 \\
\hline Control Group & $7.030 \pm 0.601$ & $5.072 \pm 0.963$ & $5.557 \pm 1.390$ & $4.123 \pm 0.882$ & $6.251 \pm 0.588$ & $6.671 \pm 0.636$ \\
\hline Model Group & $5.026 \pm 0.531^{*}$ & $4.741 \pm 0.700$ & $5.551 \pm 1.105$ & $4.729 \pm 0.091$ & $4.559 \pm 0.401^{*}$ & $4.599 \pm 0.144^{*}$ \\
\hline Acupun cture Group & $5.948 \pm 0.320^{*} \triangle$ & $7.449 \pm 0.756^{*} \triangle$ & $7.646 \pm 0.491 * \triangle$ & $7.631 \pm 0.562 * \triangle$ & $5.830 \pm 0.132 \triangle$ & $6.218 \pm 0.249 \triangle$ \\
\hline
\end{tabular}

*mean that using control group as a reference AND $\triangle$ represented that using model group as a reference

Table 2: The temporal effects on the pain threshold of control group.

\begin{tabular}{|c|c|c|c|c|c|c|}
\hline \multicolumn{1}{|c|}{ Tests of Within-Subjects Effects in the Control Group } \\
\hline \multirow{2}{*}{ Factor 1 } & Source & Type III Sum of Squares & df & Mean Square & F & Sig. \\
\hline \multirow{2}{*}{ Error (Factor 1) } & Sphericity & 35.245 & 5 & 7.049 & 7.984 & 0 \\
\cline { 2 - 8 } & Greenhouse-Geisser & 35.245 & 2.045 & 17.234 & 7.984 & 0.008 \\
\cline { 2 - 8 } & Sphericity & 22.071 & 25 & 0.883 & & \\
\hline
\end{tabular}

There was significant difference for pain threshold at different times in control group. ( $F=7.984, P=0.008, P<0.01)$.

Table 3: The temporal effects on the pain threshold of model group.

\begin{tabular}{|c|c|c|c|c|c|}
\hline \multicolumn{5}{|c|}{ Tests of Within-Subjects Effects in the Model Group } \\
\hline & Source & Type III Sum of Squares & df & Mean Square & F \\
\hline
\end{tabular}


Journal of Complementary Medicine \& Alternative Healthcare

\begin{tabular}{|c|c|c|c|c|c|c|}
\hline Factor 1 & Sphericity & 4.165 & 5 & 0.833 & 2.295 & 0.076 \\
\hline Error (Factor 1) & Sphericity & 9.073 & 25 & 0.363 & & \\
\hline
\end{tabular}

There was no significant difference at different times in model group. $(\mathrm{P}=0.076, \mathrm{P} \triangle 0.05)$.

Table 4: The temporal effects on the pain threshold of acupuncture group.

\begin{tabular}{|c|c|c|c|c|c|c|}
\hline \multicolumn{7}{|c|}{ Tests of Within-Subjects Effects in the Acupuncture Group } \\
\hline & Source & Type III Sum of Squares & df & Mean & F & Sig. \\
\hline Factor 1 & Sphericity & 22.988 & 5 & 4.598 & 24.757 & 0 \\
\hline Error (Factor 1) & Sphericity & 4.643 & 25 & 0.186 & & \\
\hline
\end{tabular}

There was significant difference at different times in the acupuncture group. $(F=24.757, P<0.01)$.

Table 5: Comparison of interaction in different groups.

\begin{tabular}{|c|c|c|c|c|c|}
\hline \multicolumn{5}{|c|}{ Tests of Within-subjects Effects } \\
\hline & Type III Sum of Squares & df & Mean Square & F & Sig. \\
\hline Source & 3649.146 & 1 & 3649.146 & 10094.38 & 0 \\
\hline Intercept group & 66.358 & 2 & 33.179 & 91.781 & 0 \\
\hline Error & 5.423 & 15 & 0.362 & & \\
\hline
\end{tabular}

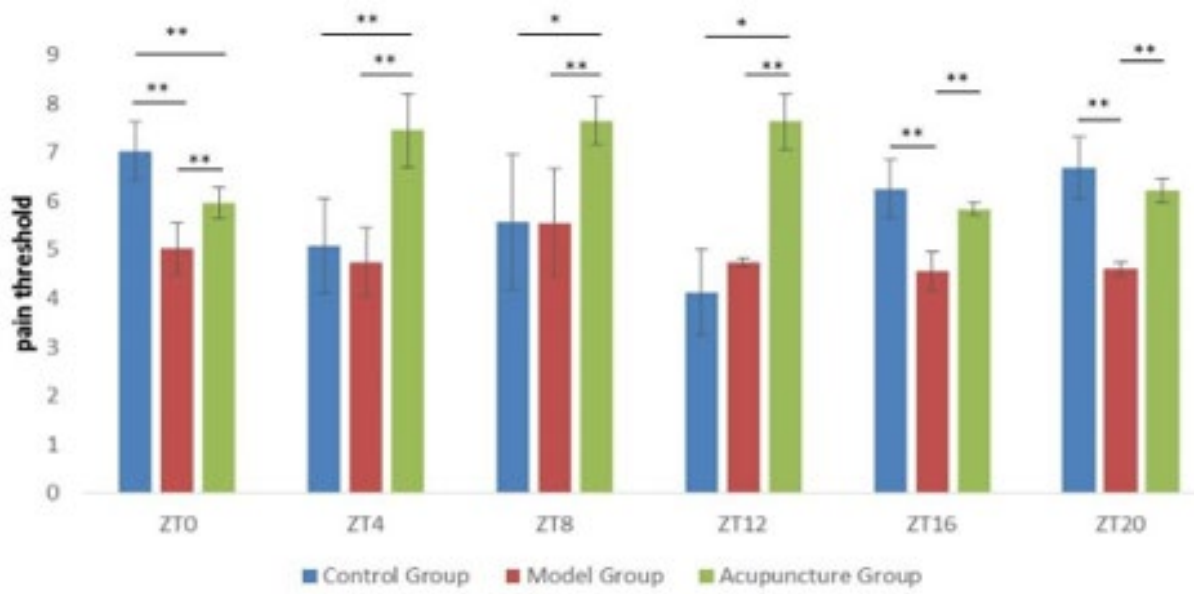

Figure1: Colored boxes indicated Control Group, Model Group, Acupuncture Group.

The pain threshold values were shown as mean $\pm \mathrm{SD}, \mathrm{N}=6$. Statistical significance comparing peak expression values was determined by Welch's t-test, shown as ${ }^{*} p<0.05,{ }^{* *} p<0.01$

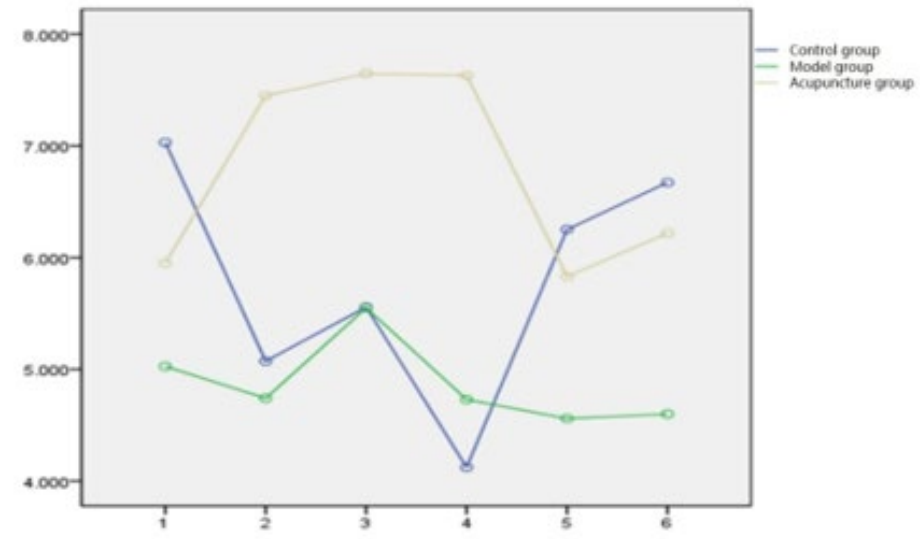

Figure2: Axis of abscissa: time1-ZT0, time2-ZT4, time3-ZT8, time4-ZT12, time5-ZT16, time6- ZT20. 
Time factor had effects on extracellular ATP concentration in different groups. There was significant difference in the variation of extracellular ATP concentration among control group, model group and acupuncture group $(\mathrm{P}<0.05)$. There was statistical significance between control group and model group at ZT12 $(\mathrm{P}=0.017, \mathrm{P}<0.05)$. Extracellular ATP concentration in acupuncture group was higher than that in model group at all six time points. However, there was only statistical significance at $\mathrm{ZT} 16(\mathrm{P}=0.022, \mathrm{P}<0.05)$. In the same group at different time points, extracellular ATP concentration also had the differences. In the control group, the extracellular ATP concentration of ZT8(P=0.016, $\mathrm{P}<0.05)$ and ZT12 $(\mathrm{P}=0.038, \mathrm{P}<0.05)$ were significantly different from that of ZT4 respectively. In the model group, the extracellular ATP concentration of $\mathrm{ZT} 4(\mathrm{P}=0.026$, $\mathrm{P}<0.05)$ and ZT20 $(\mathrm{P}=0.030, \quad \mathrm{P}<0.05) \quad$ were significantly different from that of ZT0 respectively. In the model group, the extracellular ATP concentration of ZT4(P=0.010, $\mathrm{P}<0.05)$ and ZT20 $(\mathrm{P}=0.029, \mathrm{P}<0.05)$ were significantly different from that of ZT12 respectively. And the increase of extracellular ATP concentration in acupuncture group was affected by the time factor, which was statistically significant compared with other two groups Table 6- Table 9 Figure 3 and Figure 4. The trend of extracellular ATP concentration over time. In control group, two valley value appeared at ZT4 and ZT12 and two peak value appeared at ZT0 and ZT8. Extracellular ATP concentration was in a low level at ZT12 and began to show an upward trend at ZT16. In model group, that showed one valley value and one peak value, which was ZT4 and ZT8 respectively. Compared with control group, extracellular ATP concentration abnormally increased at ZT8 and the valley value disappeared at ZT12. The trend of acupuncture group was similar to that of control group. Two valley value appeared at ZT4 and ZT12 and two peak value appeared at ZT0 and ZT8. The time of high point and the low point of extracellular ATP concentration appeared to be normal. The extracellular ATP concentration in acupuncture group was higher than that in model group.

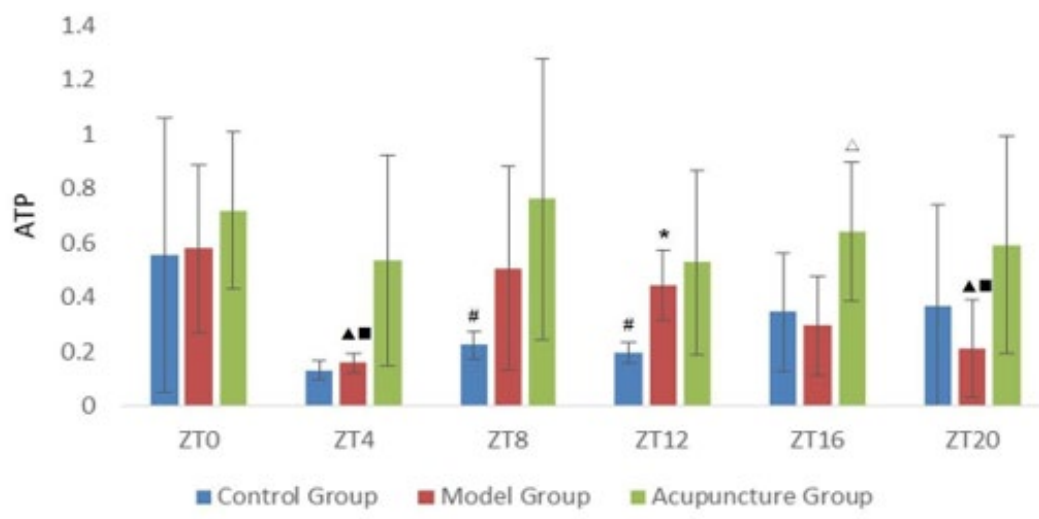

Figure 3: $\triangle P<0.05$, compared to the $\mathrm{ZT} 12$ control group; $\triangle \mathrm{P}<0.05$, compared to the $\mathrm{ZT} 16$ model group; \#P<0.05, compared to the $\mathrm{ZT} 4$ control group; $\Delta \mathrm{P}<0.05$, compared to the ZT0 model group; $\triangle \mathrm{P}<0.05$, compared to the $\mathrm{ZT} 12$ model group.

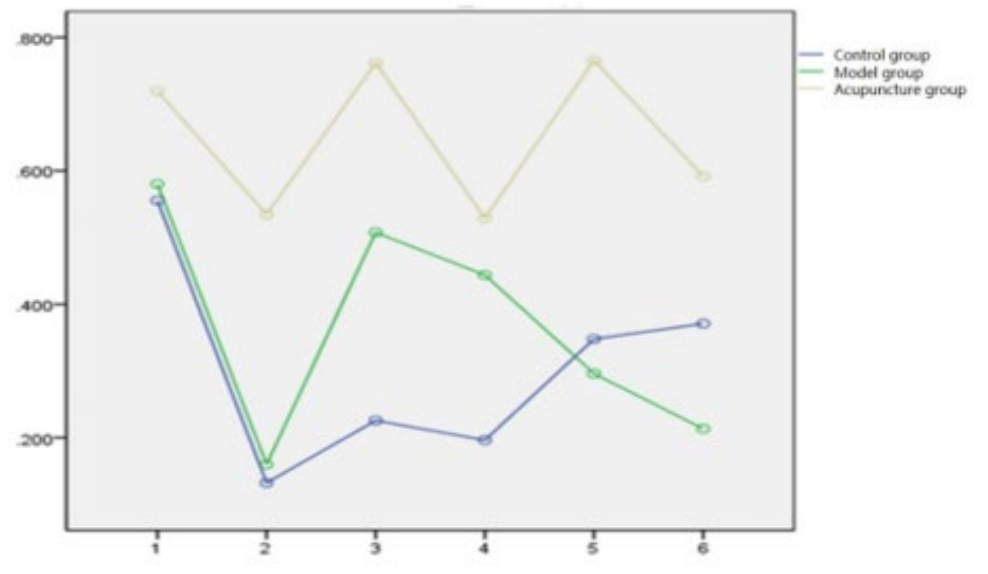

Figure 4: Axis of abscissa: time1-ZT0, time2-ZT4, time3-ZT8, time4-ZT12, time5-ZT16, time6-ZT20. 
Table 6: Data analysis for extracellular ATP concentration in local acupoints at different times points $\mathrm{n}=6$ ( $\mu \mathrm{g} / \mathrm{ml})$.

\begin{tabular}{|c|c|c|c|c|c|c|}
\hline & ZT0 & ZT4 & ZT8 & ZT12 & ZT16 & ZT20 \\
\hline Control Group & $0.555 \pm 0.503$ & $0.132 \pm 0.035$ & $0.226 \pm 0.050 \#$ & $0.196 \pm 0.038 \#$ & $0.348 \pm 0.217$ & $0.371 \pm 0.367$ \\
\hline Model Group & $0.580 \pm 0.307$ & $0.160 \pm 0.036 \mathbf{\square}$ & $0.508 \pm 0.376$ & $0.444 \pm 0.130 *$ & $0.296 \pm 0.181$ & $0.213 \pm 0.178 \mathbf{\Delta}$ \\
\hline Acupuncture Group & $0.719 \pm 0.287$ & $0.535 \pm 0.388$ & $0.762 \pm 0.516$ & $0.529 \pm 0.339$ & $0.642 \pm 0.257 \triangle$ & $0.592 \pm 0.400$ \\
\hline
\end{tabular}

* mean that using ZT12 of control group as a reference

$\triangle$ mean that using ZT16 of model group as a reference

\# mean that using ZT4 of control group as a reference

$\Delta$ mean that using ZTO of model group as a reference

- mean that using ZT12 of model group as a reference

Table 7: Sphericity test for extracellular ATP concentration in different groups.

\begin{tabular}{|c|c|c|c|c|}
\hline \multicolumn{5}{|c|}{ Mauchly's Test of Sphericity $^{\mathbf{b}}$} \\
\hline Within Subjects Effect & Mauchly's W & Approx. Chi- Square & df & Sig. \\
\hline Factor 1 & 0.322 & 14.834 & 14 & 0.398 \\
\hline
\end{tabular}

Based on the sphericity test, no correction was required $\mathrm{P}=0.398, \mathrm{P}>0.05$.

Table 8: Comparison of extracellular ATP concentration at different times.

\begin{tabular}{|c|c|c|c|c|c|c|}
\hline \multicolumn{7}{|c|}{ Tests of Within-Subjects Effects } \\
\hline & Source & Type III Sum of Squares & df & Mean Square & F & Sig. \\
\hline Factor 1 & Sphericity & 1.222 & 5 & 0.244 & 2.507 & 0.037 \\
\hline Error (Factor 1) & Sphericity & 7.313 & 75 & 0.098 & & \\
\hline
\end{tabular}

Time factor had effect on extracellular ATP concentration. There was difference in extracellular ATP concentration at different times $\mathrm{P}=0.037$, $\mathrm{P}<0.05$.

Table 9: Comparison of extracellular ATP concentration in different groups.

\begin{tabular}{|c|c|c|c|c|c|}
\hline \multicolumn{5}{|c|}{ Tests of Within-Subjects Effects } \\
\hline Source & Type III Sum of Squares & df & Mean Square & F & Sig. \\
\hline Intercept & 20.971 & 1 & 20.971 & 284.704 & 0 \\
\hline Group & 2.445 & 2 & 1.222 & 16.594 & 0 \\
\hline Error & 1.105 & 15 & 0.074 & & \\
\hline
\end{tabular}

There was significant difference in extracellular ATP concentration among different groups, $P<0.05$.

Table 10: Model R-squared.

\begin{tabular}{|c|c|c|c|c|}
\hline \multicolumn{5}{|c|}{ Model Summary $^{\mathbf{b}}$} \\
\hline Model & $\mathrm{R}$ & $\mathrm{R}^{2}$ & Adjusted R $^{2}$ & Std. Error \\
\hline 1 & $.396^{\mathrm{a}}$ & 0.157 & 0.133 & 1.173647 \\
\hline
\end{tabular}

a. Predictors: (constant), group, ATP

b. Dependent variable: pain threshold

Table 11: Significance of Regression Model.

\begin{tabular}{|c|c|c|c|c|c|c|}
\hline \multicolumn{7}{|c|}{ Anova $^{\mathbf{b}}$} \\
\hline & Model & Square & df & Mean Square & F & Sig. \\
\hline 1 & Regression & 26.712 & 3 & 8.904 & 6.464 & $.000 \mathrm{a}$ \\
\hline & Residual & 143.254 & 104 & 1.377 & & \\
\hline & Total & 169.966 & 107 & & & \\
\end{tabular}

a. Predictors: (Constant), Group, ATP

b. Dependent variable: pain threshold

Table 12: Factor correlation of influencing pain threshold.

\begin{tabular}{|l|c|c|c|c|c|}
\hline \multicolumn{5}{|l|}{ Coefficients } & \\
\hline & Unstandardized Coefficients & & Standardized Coefficients & & \\
\hline
\end{tabular}




\section{Journal of Complementary Medicine \& Alternative Healthcare}

\begin{tabular}{|c|c|c|c|c|c|c|}
\hline & Model & B & Std. Error & t & Sig. \\
\hline 1 & (Constant) & 4.953 & 0.37 & & 13.391 & $0.000 \mathrm{a}$ \\
\hline & ATP & 0.816 & 0.36 & 0.224 & 2.267 & 0.025 \\
\hline & Group & 0.361 & 0.152 & 0.235 & 2.377 & 0.019 \\
\hline
\end{tabular}

a. Dependent Variable: pain threshold

The pain threshold was mainly affected by extracellular ATP concentration in local acupoints and showed a significant positive correlation $(\mathrm{P}<0.05)$ Table 10- Table 12.

The Relationships Between Time Factor and P2X3 Receptors on Chrono-Acupuncture

As for protein expression of $\mathrm{P} 2 \mathrm{X} 3$ receptors in skin of "Zusanli" (ST36), compared with control group, it obviously increased in model group at ZT8 and ZT16. And there was significant difference between control group and model group $(\mathrm{P}<0.01)$. In addition, compared with model group, it obviously decreased after acupunctured at ZT8 and there was significant difference between model group and acupuncture group at ZT8 $(\mathrm{P}<0.01)$, but not at ZT16 $(\mathrm{P}<0.05)$. Interestingly, the protein expression of P2X3 receptors in both DRG and SDH was consistent with that in skin of "Zusanli" (ST36) Table 13- Table15, Figure 5- Figure 10.

Table 13: The effects of protein expression of P2X3 receptors in skin of Zusanli (ST36) at ZT8 and ZT16 (OD ( )).

\begin{tabular}{|l|l|l|l|l|}
\hline & N & & ZT8 & ZT16 \\
\hline Control Group & 8 & & $0.1766 \pm 0.0062$ & $0.1783 \pm 0.0047$ \\
\hline Model Group & 8 & & $0.1925 \pm 0.0055^{* *}$ & $0.1900 \pm 0.0088 * *$ \\
\hline Acupuncture Group & 8 & $0.1797 \pm 0.0085 \triangle \triangle$ & $0.1835 \pm 0.0065$ \\
\hline
\end{tabular}

Table 14: The effects of protein expression of P2X3 receptors in DRG(L4-L6) at ZT8 and ZT16 (CD ( )).

\begin{tabular}{|c|c|c|c|}
\hline & N & ZT8 & ZT16 \\
\hline Control Group & 7 & $0.2029 \pm 0.0069$ & $0.2044 \pm 0.0059$ \\
\hline Model Group & 6 & $0.2311 \pm 0.0113^{* *}$ & $0.2239 \pm 0.0076^{* *}$ \\
\hline Acupuncture Group & 8 & $0.2153 \pm 0.0118 \triangle \triangle$ & $0.2196 \pm 0.0073$ \\
\hline
\end{tabular}

Table 15: The effects of protein expression of P2X3 receptors in SDH(L4-L6) at ZT8 and ZT16 (OD ( )).

\begin{tabular}{|c|c|c|c|}
\hline & N & ZT8 & ZT16 \\
\hline Control Group & 8 & $0.1661 \pm 0.0220$ & $0.1696 \pm 0.0172$ \\
\hline Model Group & 8 & $0.2073 \pm 0.0191 * *$ & $0.2016 \pm 0.0100 * *$ \\
\hline Acupuncture Group & 8 & $0.1683 \pm 0.0246 \triangle \triangle$ & $0.1917 \pm 0.0088$ \\
\hline
\end{tabular}

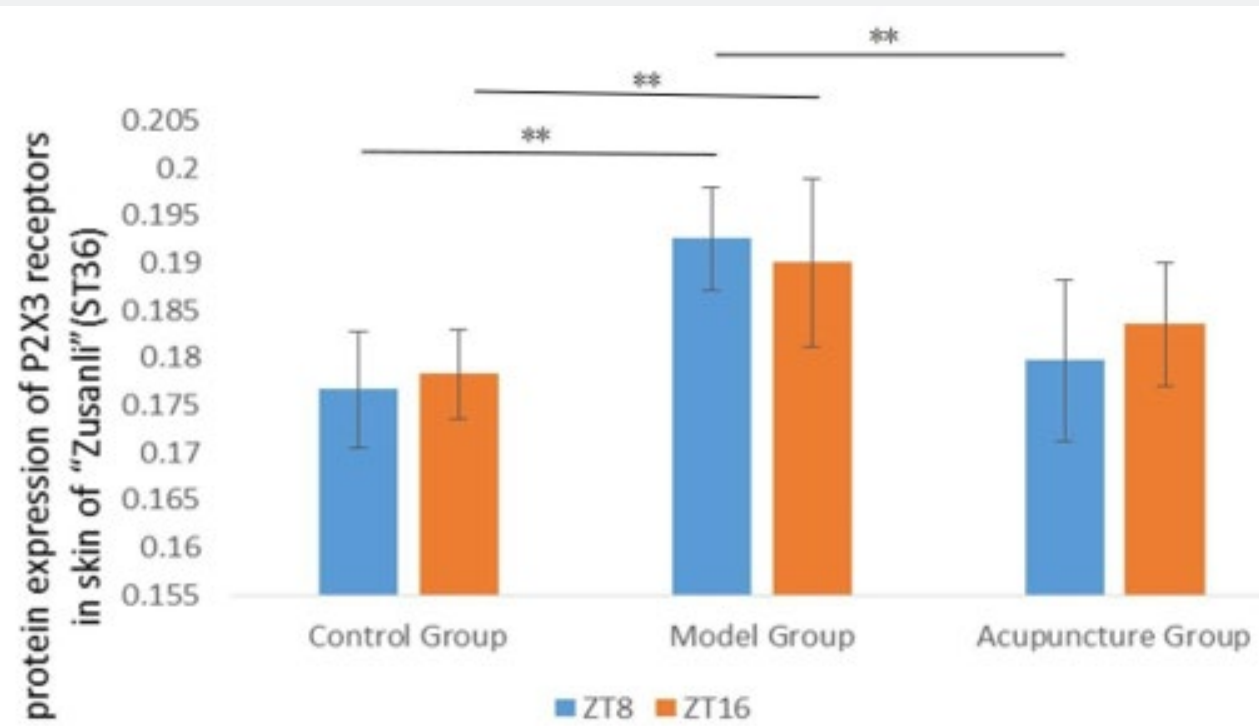

Figure 5: The effects of protein expression of P2X3 receptors in skin of "Zusanli" (ST36) values are shown as mean \pm SD, N=8. Statistical significance comparing peak expression values was determined by t-test, shown as ${ }^{* *} p<0.01$. 

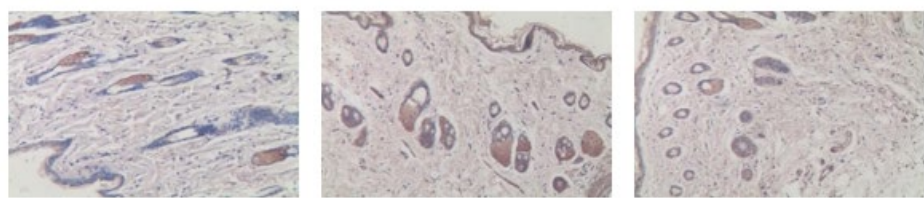

ZT8 Control Group

$(\mathrm{IHC} \times 400)$

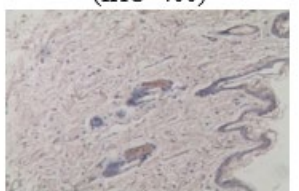

ZT16 Control Group

$(\mathrm{IHC} \times 400)$
ZT8 Model Group

$(\mathrm{IHC} \times 400)$

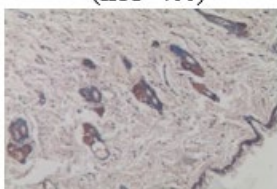

ZT16 Model Group

$(\mathrm{IHC} \times 400)$
ZT8 Acupuncture Group

$(\mathrm{IHC} \times 400)$

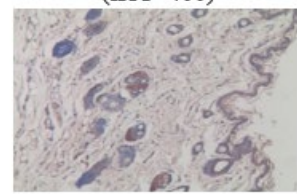

ZT16 Acupuncture Group (IHC $\times 400)$

Figure 6: The expression of P2X3 receptors in skin of "Zusanli" (ST36) at ZT8 and ZT16.

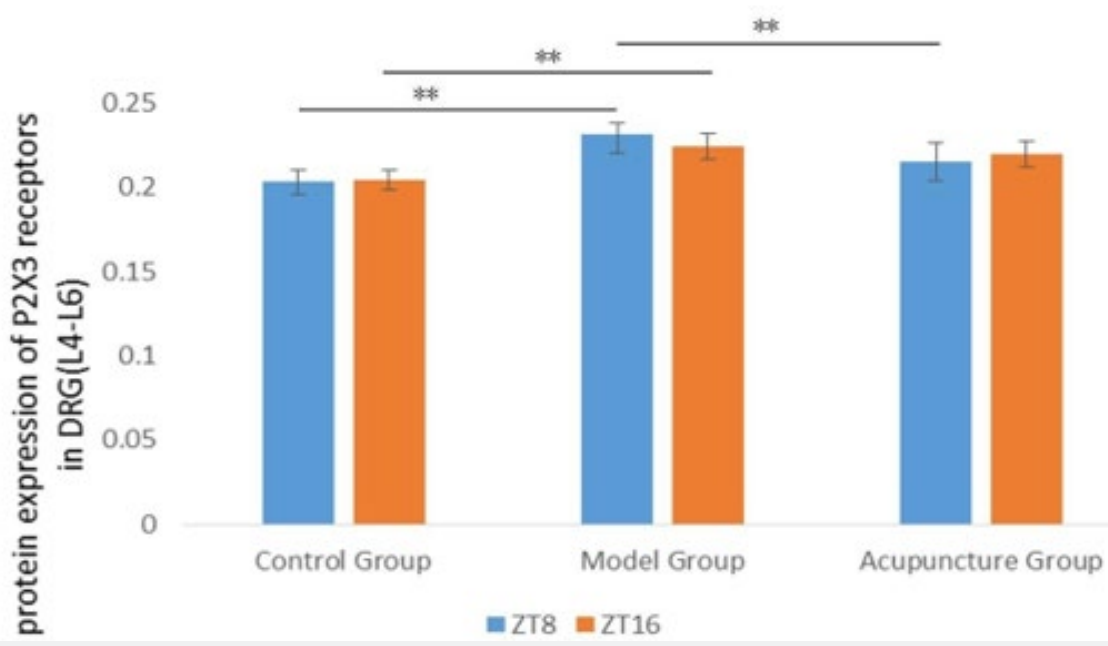

Figure 7: The effects of protein expression of $P 2 X 3$ receptors in $D R G(L 4-L 6)$ values are shown as mean $\pm S D, N=6-8$. Statistical significance comparing peak expression values was determined by t-test, shown as ${ }^{* *} \mathrm{p}<0.01$.
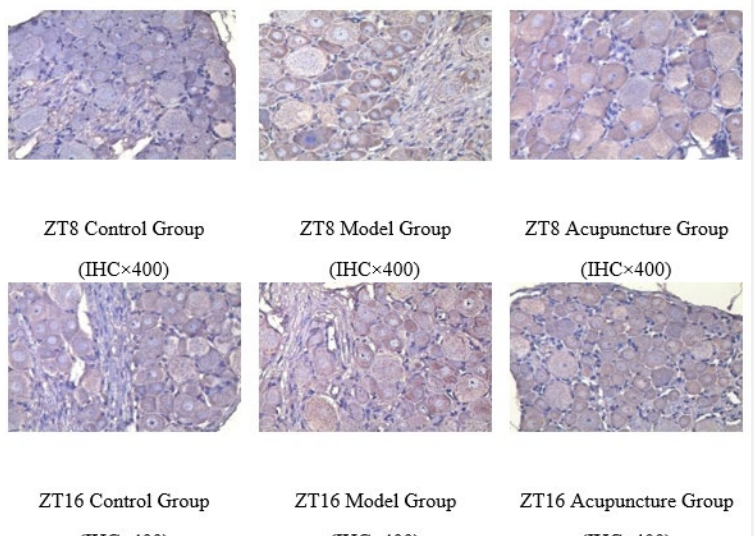

ZT16 Acupuncture Group

$(\mathrm{IHC} \times 400)$

(IHC $\times 400)$

$(\mathrm{IHC} \times 400)$

Figure 8: The expression of $\mathrm{P} 2 \mathrm{X} 3$ receptors in $\mathrm{DRG}(\mathrm{L4}-\mathrm{L} 6)$ at ZT8 and ZT16. 


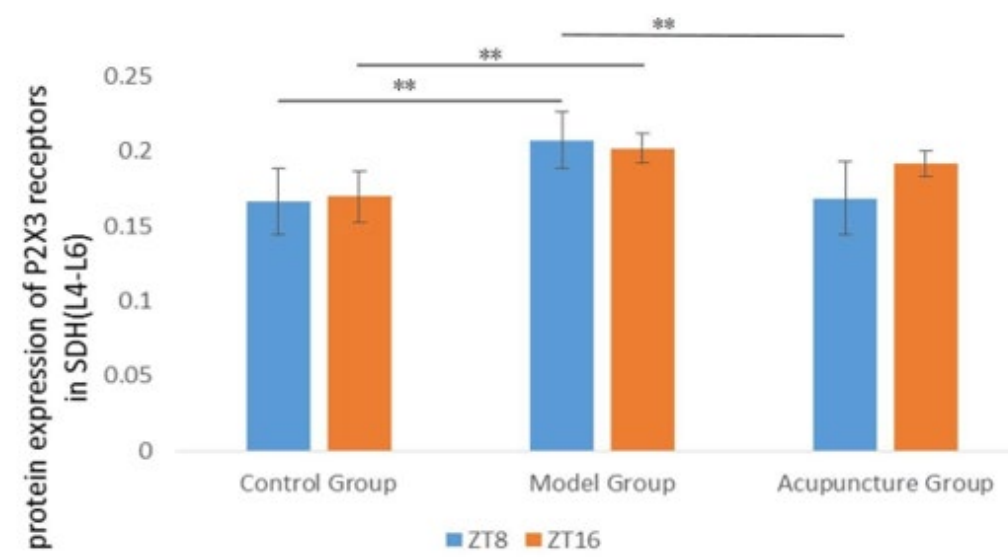

Figure 9: The effects of protein expression of $\mathrm{P} 2 \mathrm{X} 3$ receptors in $\mathrm{SDH}(\mathrm{L} 4-\mathrm{L} 6)$ values are shown as mean $\pm \mathrm{SD}, \mathrm{N}=6-8$. Statistical significance comparing peak expression values was determined by $\mathrm{t}$-test, shown as ${ }^{* *} \mathrm{p}<0.01$.
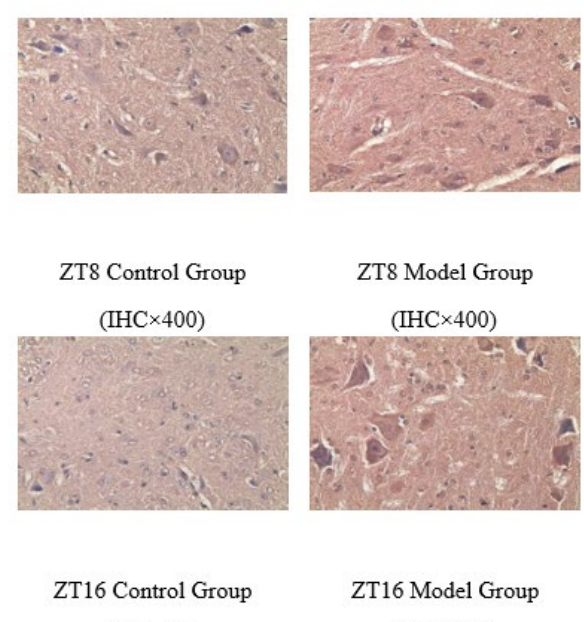

$(\mathrm{IHC} \times 400)$
ZT16 Model Group

$(\mathrm{IHC} \times 400)$

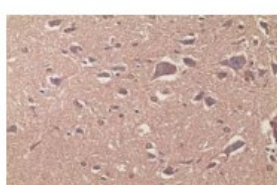

ZT8 Acupuncture Group

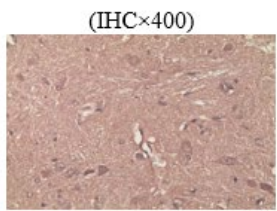

ZT16 Acupuncture Group

$(\mathrm{IHC} \times 400)$

Figure 10: The expression of $\mathrm{P} 2 \mathrm{X} 3$ receptors in $\mathrm{SDH}(\mathrm{L} 4-\mathrm{L} 6)$ at $\mathrm{ZT} 8$ and $\mathrm{ZT} 16$.

\section{Discussion}

This work indicated that time factor was an important regulator of purinergic signaling in acupuncture analgesia. We found that:

1. Acupuncture not only increased pain threshold but had the function of temporal regulation on pain threshold.

2. The variation of extracellular ATP concentration in local acupoints was affected by acupuncture in different time points and there was a positive correlation between pain threshold and extracellular ATP concentration.

3. The variation of protein expression of $\mathrm{P} 2 \mathrm{X} 3$ receptors in local points, DRG and SDH had temporal difference after acupunctured at different time points.

Together, our results showed that, in the peripheral mechanism, the acupoint reactivity of chrono- acupuncture analgesia might be related to temporal changes of purinergic signaling in local acupoints. Both master clock and peripheral clock could modulate the circadian rhythm of lives according environment time cues. It was found that the mechanism of molecular oscillations in the peripheral biological clock was similar to that of the master clock [17]. If there were abnormalities in circadian rhythm of the body, chrono-biological indicators changed accordingly. There were some special rhythm characters in many kinds of diseases, especially in pain syndromes. As for rodents, the responses of pain were drastic in dark and moderate in light [18-19]. In clinical studies, pain intensity and body response had rhythmic and cyclical changes. Multiple signaling involved in the rhythmic changes in pain syndromes [20]. Our findings indicated that acupuncture not only increased the inflammatory pain threshold in rats, but also the acupuncture-time interaction had a significant effect on the pain threshold. At different time points, the peak value of pain threshold in control group appeared at ZT0 and the valley value appeared at ZT12. However, the rhythms of animal pain changed after model establishment. The pain threshold of model group was significantly lower than that of control group at ZT0, ZT16 
and ZT20 respectively, except at ZT12. Additionally, the peak value of pain threshold in acupuncture group appeared at ZT8 and the valley value appeared at ZT16. Compared with model group, the pain threshold of acupuncture group at the same time point was upward generally. On the whole, time factor was markedly effect on three groups in this experiment. The interaction had significant influences on the pain threshold. Based on experimental results, the pain threshold of rats was rhythmic in physiological conditions. While the pain threshold had abnormal rhythm changes after establishment. And acupuncture could increase the inflammatory pain threshold in rats. The interaction of acupuncture and time factor had significant effect on the pain threshold. In other words, the changes of pain threshold aroused by acupuncture at different times was significantly different. Studies focusing on the extracellular purinergic signaling, represented by ATP, was important informational substances in the body's temporal structure. In both peripheral and central oscillatory system, extracellular ATP and its metabolites showed marked circadian rhythm. Under the cycle of LD12:12 and DD, the extracellular ATP level in SCN neurons of rats had obvious rhythmical changes. While the extracellular ATP accumulation in vitro cultured cortical glial cells also showed diurnal oscillations [21-22]. The circadian variation of ATP hydrolase activity was of importance for maintaining the temporal function of extracellular purinergic signaling. ATP hydrolase activity in peripheral blood also had rhythmical change, and the highest reactivity occur in dark period [23]. Furthermore, extracellular ATP could selectively raise the expression of gene mPer1 to affect the basic working pattern which was transcription- translation negative feedback among molecular clocks by activating P2X7 receptor [24]. So, extracellular ATP not only outputted temporal information, but also had the function of temporal modulation. Our findings indicated that, the extracellular ATP concentration in control group had double peak (ZT0 and ZT8) and double valley (ZT4 and ZT12). The extracellular ATP concentration of ZT12 was at a low level and began to increase at ZT16. However, the model group showed only one peak (ZT8) and one valley (ZT4). Compared with control group, there was an abnormal increase at ZT8 and the original valley at ZT12 disappeared. The variation of acupuncture group is similar to that of control group and showed double peak (ZT0 and ZT8) and double valley (ZT4 and ZT12). The peak and valley value of extracellular ATP concentration tended to be normal. And the extracellular ATP concentration in local appoints in acupuncture group was higher than that of model group. Combined with pain threshold and extracellular ATP concentration, our results showed that the pain threshold was mainly affected by extracellular ATP concentration in local acpoints and shows positive correlation. There was also a positive correlation between processing methods (whether the rats were in healthy conditions or not and whether acupuncture was adopted).

The studies showed that ATP involved in the pain conduction. P2X3 receptors, a non-selective ligand gated cation channel, played a key role in peripheral transmission of pain [12]. ATP could induce mechanical hyperalgesia in gastrocnemius of rats to release proinflammatory cytokine by activation of peripheral P2X3 receptor [25]. ATP was released from injured cells and excited peripheral P2X3 and P2X3 receptors to generate action potentials of primary afferent neurons, transmit them to the distal end of the central nervous system and released the neurotransmitter's effect on the postsynaptic process of the central nervous system Neurons [26]. Related studies had shown that P2X3 receptor-induced pain could be significantly reduced after blocking the $\mathrm{P} 2 \mathrm{X} 3$ receptors' conduction pathway or using P2X3 receptor antagonists. After sciatic nerve was cut off, the expression of P2X3 receptor in L4/5 DRG decreased by 50\% [27]. It had found that, TNP-ATP, a P2X1, P2X3 and P2X2/3 receptor antagonist, significantly attenuates inflammatory hyperalgesia in the rat model of inflammatory pain induced by carrageenan in the temporomandibular joint (TMJ). Whereas $\mathrm{P} 2 \mathrm{X} 1$ receptor did not participate in this model pain, which mean that P2X3 and $\mathrm{P} 2 \mathrm{X} 2 / 3$ receptor might involve in the inflammatory pain sensitivity of the rat model of TMJ [28]. A-317491, a selective P2X3 receptor antagonist, could reduce chronic inflammatory and neuropathic pain mediated by P2X3 receptor in rats. And intrathecal administration of A-317491 seemed to be more effective to relieve tactile allodynia after peripheral nerve injury than intraplantar administration [29]. Meanwhile, many

studies showed that, purinergic signaling played an important role in acupuncture analgesia. Electro-acupuncture could exert an analgesic effect on neuropathic pain through simultaneous action of purine $\mathrm{Al}$ and $\mathrm{P} 2 \mathrm{X} 3$ receptors [30]. Also, acupuncture could reduce the expression of $\mathrm{P} 2 \mathrm{X} 3$ receptor gene and the magnitude of ATP excitation current in DRG of rats with chronic constriction injury (CCI) [31]. What's more, midbrain periaqueductal gray $\mathrm{P} 2 \mathrm{X} 3$ receptors involved in the modulation mechanism of electro-acupuncture analgesia in the spinal cord. ATP had circadian rhythm and had the function of temporal modulation [32]. Our findings indicated that, no matter in skin of "Zusanli" (ST36), DRG and SDH, compared with control group, the protein expression of P2X3 receptors in model group markedly increases at ZT8 and ZT16 respectively. While compared with model group, the protein expression of P2X3 receptors in acupuncture group markedly decreases at ZT8, but not at ZT16. These results mean that, there was temporal difference in decreasing the protein expression of P2X3 receptors by acupuncture. Moreover, after both establishing and acupuncturing, the variation of protein expression of P2X3 receptors in DRG and SDH displayed highly consistency at the same time point. This might be one of the foundations of the time effect of acupuncture analgesia.

\section{Experimental Procedures}

\section{Animals}

The experiment consisted of two parts. 156 SD male rats were needed in this experiment. All rats (body weight of 200 
\pm 20 g) were provided by animal center of Sichuan provincial hospital (Permit Number: SCXK(CHUAN)2013-15). The rats were acclimated to the rat facility for 1 week before starting the experiments. The rats were housed under $12 \mathrm{~h}: 12 \mathrm{~h}$ light/ dark cycles (Light on at 7:00, ZT0 and off at 19:00, ZT12) with free access to water during the whole experiment, and free access to food. Animal care was carried out in accordance with the Instruction for Ethical Treatment of Animals issued by the Ministry of Science and Technology, China, in 2006. We tried to minimize the number and suffering of the laboratory animals. All procedures and animal experiments were approved by the Animal Care and Use Committee of Chengdu University of Traditional Chinese Medicine (China) (N0.2014-01).

\section{Assessment of basic pain threshold}

The basic pain threshold of rats was tested by tail-flick method. The standard rats which tailed between 3 and 10 seconds on the test were involved in the experiment. The basic pain threshold was needed to finish within 1 hour under the same condition. In addition, in order to avoid scalding the rats and calm them down, each one was measured at intervals of 5 minutes or more.

\section{Grouping and modeling}

In this part experiment,108 SD male rats were randomized divided into two parts. One part was subcutaneously injected $0.1 \mathrm{ml} 0.9 \%$ saline into the right hind paw for 1 day before the experiment as control group. Under the same condition, another part was subcutaneously injected $0.1 \mathrm{ml}$ CFA into the right hind paw for 1 day before the experiment. After successful replication of the model, it was randomly divided into model group and acupuncture group based on the basic pain threshold. And each group was divided into 6 sub-groups which were ZT0, ZT4, ZT8, ZT12, ZT16 and ZT20 respectively. The rats established by CFA should be in acute pain stage after 24 hours of injection and the syndromes showed that the time of tail-flick shortened accompanying with red-swollen paw.

\section{Experimental progress}

Assessment of pain threshold: At the second day after model establishment, the rats in acupuncture group acupunctured "Zusanli" (ST36) in the affected side for 30 min (twisted 1 min every $5 \mathrm{~min}, 120 \mathrm{per} / \mathrm{min}$ ). When the therapy was finished, the pain threshold of rats was tested by tail-flick method. The pain threshold was needed to finish within 1 hour under the same condition. Additionally, in order to avoid scalding the rats and calm them down, each one was measured at intervals of 5 minutes or more.

Assessment of extracellular ATP concentration in local acupoint: We collected samples of interstitial fluid by a micro dialysis probe implanted in the tibialis anterior muscle/sub cutis of rats at a distance of 0.4-0.6 mm from "Zusanli" (ST36). The processes of this experiment as follows:

1. Under the constant temperature of $37^{\circ} \mathrm{C}$ with a CMA/450 animal thermostat, the rats were anesthetized with $10 \%$ chloral hydrate $(0.4 \mathrm{ml} / 100 \mathrm{~g})$ by intraperitoneal injection and kept them in prone position. The right hind leg of the rats was shaved, disinfected and kept operating area clean and dry.

2. The length of the catheter was guided by micro dialysis probe accurately.

3. Cut off the skin where the probe had been marked with tissue scissors and tissue forceps. The wound was minimally invasive as long as the probe was successfully inserted. The syringe needle was needed to insert into the tear supporting tube and was slowly implanted local tissue of acupoints. Then, the syringe needle was put out, and the micro dialysis probe (CMA20, Sweden) was inserted into the tear supporting tube.

4. The two ends of the probe were respectively connected to the microanalysis pump (CMA402) and the cooling microcollector (MAB85). In the micro dialysis pump, 0.9\% saline was injected into the injector, with the speed of $1 \mu \mathrm{L} / \mathrm{min}$ and balanced for 1 hour. In the cooling micro-collector, the temperature was set to $4^{\circ} \mathrm{C}$. After the balance, the local tissue fluid of the acupoints was collected by the cooling microcollector automatically (30min / 1 sample). Each rat was collected for two samples which were respectively before and during acupuncture. The sample collections lasted for 1 hour.

5. The content of purine in the sample collected by the microanalysis was tested by HPLC.

Assessment of protein expression of $\mathrm{P} 2 \mathrm{X} 3$ receptors in skin of "Zusanli" (ST36), DRG and SDH: Based on the former experimental results, the peak value (ZT8) and the valley value (ZT16) were chosen for the further experiment. 48 SD male rats were needed in this part experiment. The methods of assessment of basic pain threshold, modeling and grouping were the same as the former. The rats in acupuncture group acupunctured "Zusanli" (ST36) in the affected side for $30 \mathrm{~min}$ (twisted $1 \mathrm{~min}$ every $5 \mathrm{~min}, 120$ per/min). Meanwhile, the rats in the control group and model group did not accept acupuncture therapy. When the therapy was finished, the rats were anesthetized with $10 \%$ chloral hydrate $(0.4 \mathrm{ml} / 100 \mathrm{~g})$ by intraperitoneal injection and the skin and subcutaneous tissue of "Zusanli" (ST36) in the affected side (size:2*2; depth:2 3mm ), DRG(L4-L6) and SDH (L4-L6) were respectively took away, then immediately placed in $4 \%$ paraformaldehyde and examined by immunohistochemistry and average optical density method.

\section{Statistical Analysis}

All data were analyzed by SPSS19.0 and results were presented as mean \pm standard deviation (SD). Statistical methods which consisted of one-way ANOVA (one-way analysis of variance), RMANOVA (repeated measures analysis of variance) and MANOVA (multivariate analysis of variance) were used to compare between the groups after the normal distribution 
test and the homogeneity test of varuances. Significance was determined at $\mathrm{P}<0.05$.

\section{Acknowledgment}

This study was funded by National Natural Science Foundation of China (NO. 628313). The authors have declared that no competing interests exist.

\section{Author Contribution}

Conceived and designed the experiments: DC ZZ. Performed the experiments: XL XS SH YW XZ NL. Analyzed the data: QZ JZ. Wrote the manuscript: SH XL XS NL.

\section{References}

1. Mohawk JA, Green CB, Takahashi JS (2012) Central and peripheral circadian clocks in mammals. Annual Review of Neuroscience 35(1): 445-462.

2. Cai DJ, Wei JL, Zhao JL (2011) Research on phase characteristic of acupuncture on circadian rhythm disturbance due to advanced light/dark cycle. China Journal of Traditional Chinese Medicine and Pharmacy 26(4): 691-694.

3. Levi F, Schibler U (2007) Circadian rhythms: mechanisms and therapeutic implications. Annual Review of Pharmacology and Toxicology 47(47): 593-628.

4. Van Oosterhout W, Van Someren E, Schoonman GG, Louter MA, Lammers GJ, et al. (2017) Chronotypes and circadian timing in migraine. Cephalalgia 38(4): 617-625.

5. Jankowski KS (2013) Morning types are less sensitive to pain than evening types all day long. Eur J Pain 17(7): 1068-1073.

6. Costa Martins JM, Pereira M, Martins H, Moura Ramos M, Coelho R, eta l. (2014) The influence of women's attachment style on the chronobiology of labour pain, analgesic consumption and pharmacological effect. Chronobiology International 31(6): 787-796

7. Chang KH, Bai SJ, Lee H, Lee BH (2014) Effects of acupuncture stimulation at different acupoints on formalin-induced pain in rats. Korean Journal of Physiology \& Pharmacology Official Journal of the Korean Physiological Society \& the Korean Society of Pharmacology 18(2): 121-127.

8. Han JS (2011) Acupuncture analgesia: areas of consensus and controversy. Pain 152(3 Suppl): S41-S48.

9. Yao HJ, Song HT, Mo YP, Zhang TT, Han XB, et al. (2014) Effects of electroacupuncture on circadian rhythm of temperature and melatonin in depression rats model induced by chronic stress. Chinese Acupuncture \& Moxibustion 34(7): 685-689.

10. Xiao Hong LI, Song XL, Li Li XU, Zhang LF, Guo MW, et al. (2010) Study of $\beta$-ep content and hsp7 0 expression in hypothalamus and pituitary of dysmenorrhea rats with acupuncture on sanyinjiao point at different time. China Journal of Traditional Chinese Medicine \& Pharmacy.

11. Wang F, Jiang Fan Chen, Jurgen Schnermann, Kim Tieu, Lane Bekar, et al. (2010) Adenosine A1 receptors mediate local anti-nociceptive effects of acupuncture. Deutsche Zeitschrift Für Akupunktur 13(7): 883-888.

12. Wang L, Feng D, Yan H, Wang Z, Pei L (2014) Comparative analysis of $\mathrm{p} 2 \times 1, \mathrm{p} 2 \times 2, \mathrm{p} 2 \mathrm{x} 3$, and $\mathrm{p} 2 \times 4$ receptor subunits in rat nodose ganglion neurons. Plos One 9(5): e96699.

13. Xu GY, Huang LY (2002) Peripheral inflammation sensitizes p2x receptor-mediated responses in rat dorsal root ganglion neurons. Journal of Neuroscience the Official Journal of the Society for Neuroscience 22(1): 93-102.
14. Mo G, Stephen Z, Jennifer L, Martin P (2006) Functional changes of P2X3 and P2X2/3 receptors in dissociated small DRG neurons under neuropathic condition induced by spinal nerve ligation in rats. International Pharmacological Assembly. pp:112.

15. Chen L, Liu YW, Yue K, Ru Q, Xiong Q et al. (2016) Differential expression of ATP-gated p2x receptors in DRG between chronic neuropathic pain and visceralgia rat models. Purinergic Signal 12(1): 79-87.

16. Tu WZ, Cheng RD, Cheng B, Lu J, Cao F, et al. (2012) Analgesic effect of electroacupuncture on chronic neuropathic pain mediated by p2x3 receptors in rat dorsal root ganglion neurons. Neurochemistry International 60(4): 379-386.

17. David K Welsh, Seung Hee Yoo, Andrew C Liu, Joseph S Takahashi, Steve A Kay (2004) Bioluminescence imaging of individual fibroblasts reveals persistent, independently phased circadian rhythms of clock gene expression. Curr biol 14(24): 2289-2295.

18. Zhou C, Petroll WM (2010) Rho kinase regulation of fibroblast migratory mechanics in fibrillar collagen matrices. Cell Mol Bioeng 3(1): 76-83.

19. Abbott RD, Koptiuch C, Iatridis JC, Howe AK, Badger GJ et al. (2013) Stress and matrix-responsive cytoskeletal remodeling in fibroblasts. Journal of Cellular Physiology 228(1): 50-57.

20. Zhang J, Li H, Teng H, Zhang T, Luo Y, et al. (2012) Regulation of peripheral clock to oscillation of substance P contributes to circadian inflammatory pain. Anesthesiology 117(1): 149-160.

21. Marpegan L, Swanstrom AE, Chung K, Simon T, Haydon PG, et al. (2011) Circadian regulation of ATP release in astrocytes. Journal of Neuroscience the Official Journal of the Society for Neuroscience 31(23): 8342-8350.

22. Womac AD, Burkeen JF, Neuendorff N, Earnest DJ, Zoran M] (2009) Circadian rhythms of extracellular ATP accumulation in suprachiasmatic nucleus cells and cultured astrocytes. European Journal of Neuroscience 30(5): 869-876.

23. Detanico BC, De Souza A, Medeiros LF, Rozisky JR, Caumo W, et al. (2010) 24-hour temporal pattern of NTPDase and $5^{\prime}$-nucleotidase enzymes in rat blood serum. Chronobiology International 27(10): $1751-1761$.

24. Nakazato R, Takarada T, Yamamoto T, Hotta S, Hinoi E, eta l. (2011) Selective upregulation of per 1 mRNA expression by ATP through activation of $\mathrm{p} 2 \times 7$ purinergic receptors expressed in microglial cells. Journal of Pharmacological Sciences 116(4): 350-361.

25. Schiavuzzo JG, Teixeira JM, Melo B, Jorge CO, Oliveira Fusaro M, et al. (2015) Muscle hyperalgesia induced by peripheral $\mathrm{p} 2 \times 3$ receptors is modulated by inflammatory mediators. Neuroscience 285: 24-33.

26. Barclay J, Patel S, Dorn G, Wotherspoon G, Moffatt S, et al. (2002) Functional downregulation of $\mathrm{p} 2 \times 3$ receptor subunit in rat sensory neurons reveals a significant role in chronic neuropathic and inflammatory pain. Journal of Neuroscience the Official Journal of the Society for Neuroscience 22(18): 8139-8147.

27. Bradbury EJ, Burnstock G, Mcmahon SB (1998) The expression of p2x3 purinoreceptors in sensory neurons: effects of axotomy and glialderived neurotrophic factor. Molecular \& Cellular Neurosciences 12(45): 256-258.

28. Teixeira JM, Oliveira MC, Clemente Napimoga JT, Pelegrini Da Silva A, Parada CA (2010) Involvement of temporomandibular joint p2x3 and $\mathrm{p} 2 \times 2 / 3$ receptors in carrageenan- induced inflammatory hyperalgesia in rats. European Journal of Pharmacology 645(1-3): 79-85.

29. Mcgaraughty S, Wismer CT, Zhu CZ, Mikusa J, Honore P, et al. (2004) Effects of A-317491, a novel and selective $\mathrm{p} 2 \times 3 / \mathrm{p} 2 \times 2 / 3$ receptor antagonist, on neuropathic, inflammatory and chemogenic nociception following intrathecal and intraplantar administration. br j pharmacol 140(8): 1381-1388 
30. Ren Wen, Tu Wenzhan, Jiang Songhe, Cheng Ruidong, Du Yaping (2012) Electroacupuncture improves neuropathic pain: adenosine, adenosine 5'-triphosphate disodium and their receptors perhaps change simultaneously. Neural Regeneration Research 7(33): 2618-2623.

31. Cheng RD, Tu WZ, Wang WS, Zou EM, Cao F, et al. (2013). Effect of electroacupuncture on the pathomorphology of the sciatic nerve and the sensitization of $\mathrm{p} 2 \mathrm{x} 3$ receptors in the dorsal root ganglion in rats with chronic constrictive injury. Chinese Journal of Integrative Medicine 19(5): 374-379.

32. Xiao Z, Ou S, He WJ, Zhao YD, Liu XH, et al. (2010) Role of midbrain periaqueductal gray $\mathrm{p} 2 \mathrm{x} 3$ receptors in electroacupuncture-mediated endogenous pain modulatory systems. Brain Research 1330(1330): 31-44.

\section{Your next submission with Juniper Publishers will reach you the below assets}

- Quality Editorial service

- Swift Peer Review

- Reprints availability

- E-prints Service

- Manuscript Podcast for convenient understanding

- Global attainment for your research

- Manuscript accessibility in different formats ( Pdf, E-pub, Full Text, Audio)

- Unceasing customer service

Track the below URL for one-step submission https://juniperpublishers.com/online-submission.php 